\title{
Minimally invasive and robotic approaches to mitral valve: Robotic is best
}

Aubrey Almeida, MBBS, FRACS,,${ }^{\mathrm{a}, \mathrm{c}, \mathrm{c}}$ Elli Tutungi, MBBS, FRANZCA, ${ }^{\mathrm{c}}$ Simon Moten, MBBS, FRACS, ${ }^{\mathrm{c}}$ and Yi Chen, MBBS, PhD, FRACS ${ }^{\mathrm{a}, \mathrm{b}}$

Video clip is available online.

Asking the question "What is the best minimally invasive mitral valve operation?" is fraught with the same problems as asking, "What is the best ... city, ...car, ...football team?" etc. It depends not only on what factors one considers important but also on how one weights each of those.

While there are some parameters in mitral valve (MV) surgery that most would agree are fundamental and commonly measured, such as mortality and rate of repair, there are others that are more nebulous. Many factors important to patients, such as recovery time, return to normal activity, cosmesis, avoidance of long-term medication, and reintervention, are frequently not considered and are difficult to measure.

The situation is further complicated by the need to accommodate not one viewpoint but multiple perspectives, such as the patient, the surgeon, and the health care system. In many circumstances, these needs are antithetical.

For many reasons, the evidence to support superiority of one surgical approach over another is profoundly limited. We are not comparing one pill with another; rather, we are comparing complex management systems with individual clinicians at the core. Because of this, surgery does not lend itself to randomized controlled studies. The biases of patient selection, pathologic complexity, and outcome definition (and many others) make comparison between case series virtually impossible.

\footnotetext{
From the ${ }^{\mathrm{a} D e p a r t m e n t}$ of Cardiothoracic Surgery, Monash Medical Centre; ${ }^{\mathrm{b}}$ Department of Surgery (School of Clinical Sciences at Monash Health), Monash University; and ${ }^{\mathrm{c} C}$ ardiac Clinical Institute, Epworth Hospital, Melbourne, Australia.

Received for publication Aug 10, 2021; accepted for publication Sept 27, 2021; available ahead of print Oct 7, 2021.

Address for reprints: Aubrey Almeida, MBBS, FRACS, Department of Cardiothoracic Surgery, Monash Medical Centre, 246 Clayton Rd, Clayton, Victoria 3168, Australia (E-mail: aubreyalmeida@hotmail.com).

JTCVS Techniques 2021;10:75-9

2666-2507

Copyright (c) 2021 The Author(s). Published by Elsevier Inc. on behalf of The American Association for Thoracic Surgery. This is an open access article under the CC BY-NC-ND license (http://creativecommons.org/licenses/by-nc-nd/4.0/).

https://doi.org/10.1016/j.xjtc.2021.09.054
}

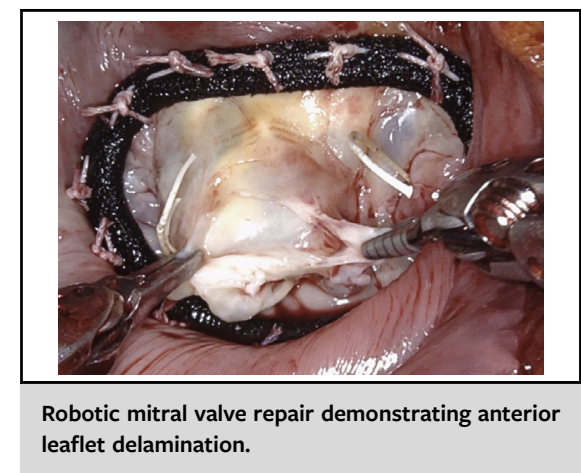

CENTRAL MESSAGE

Robotic technology facilitates sophisticated mitral valve repair via small incisions with the quickest recovery and in a manner that is most acceptable to the patient. See Commentaries on pages 80 and 82.

However, at the most basic level, irrespective of approach, the "best" MV surgery needs to prove its efficacy and safety. So how does robotic mitral surgery measure up compared with the standard approach through median sternotomy?

\section{ROBOTIC VERSUS MEDIAN STERNOTOMY APPROACH}

Over the last 20 years, several North American centers have achieved high-volume robotic mitral experience with good outcomes. In 2015, Murphy and colleagues ${ }^{1}$ reported outcomes in 1257 consecutive cases of robotic MV surgery, of which $93 \%$ were MV repair. Mortality was $0.9 \%$ and stroke in $0.7 \%$. Predischarge echocardiogram showed trace or mild mitral regurgitation (MR) in more than $98 \%$ of repaired valves and at a mean follow-up of $50 \pm 26$ months, $3.8 \%$ patients required reoperation. Gillinov and colleagues ${ }^{2}$ reported similar outcomes in their first 1000 robotic mitral procedures, with an in-hospital mortality of $0.1 \%$ and stroke rate of $1.4 \%$, which declined from $2 \%$ in the first 500 patients in the series to $0.8 \%$ in the second 500 patients. More than $99 \%$ were planned MV repair, and $97.8 \%$ of 
these had mild or less MR on the predischarge echocardiogram. These results are comparable with results achieved through sternotomy, such as reported by Castillo and colleagues, ${ }^{3}$ where more than $99 \%$ were valve repairs, with $0.8 \%$ mortality and $0.5 \%$ major stroke rate. Although there are no randomized trial data (the likelihood of there ever being such a trial is low), a meta-analysis of robotic versus sternotomy approaches showed similar overall outcomes. $^{4}$

These results demonstrate what is possible with robotic approach when it comes to efficacy and safety. Although as surgeons we have all strived to achieve the best longterm outcome for our patients, we must also consider achieving this with the quickest recovery and in a manner that is most acceptable to the patient. It is clearly the aim of minimally invasive approaches to address these needs, robotic or otherwise.

\section{ROBOTIC VERSUS OTHER MINIMALLY INVASIVE MV SURGERY}

While there is, perhaps, a gradation in minimally approaches from partial sternotomy, ${ }^{5}$ right thoracotomy with direct or thoracoscopic vision ${ }^{6}$ to robotic, with each of these there are advantages and disadvantages. Partial sternotomy MV repair is in many ways a hybrid minimally invasive approach. It is surgically more familiar than other approaches and therefore will have a shorter learning curve. However, it does come with limitations: in many, the incision is difficult to hide, and access to the MV suffers from the same limitation as through a full sternotomy.

The right thoracotomy direct vision approach with or without endoscopic assistance is probably the most widely adopted minimally invasive approach. Cosmesis is superior, especially in women (in whom the incision can be hidden in the breast crease). Thoracoscopic techniques enable a small cosmetic incision and an excellent view of the valve. Although it requires the use of peripheral cannulation and shafted instruments, the skill sets required are easily adaptable from open mitral surgery, and this has been adopted as standard of care in many large-volume centers. Indeed, a recent meta-analysis have shown similar efficacy and safety using a minimally invasive approach as compared with standard sternotomy approach.

However, due to the intrinsic limitation of the instruments, we feel this thoracoscopic approach may narrow the repertoire of mitral repair techniques used. Shafted instruments have limited freedom of movement at the tissue interface. A static left atrial (LA) retractor lacks versatility in visualizing the various components of the mitral apparatus. In addition, fundamentally, with endoscopic MV surgery, the operation is largely carried out by a single operator via shafted instruments with very little room for active assisting from a second operator. In the authors' opinion, the robotic platform overcomes the limitations of the thoracoscopic approaches.

The high-resolution 3-dimensional camera combined with the flexibility of a dynamic LA retractor affords an unsurpassed view of the valve and its components. It is simple to gain access to the subvalvular apparatus for neochord placement. The retractor may also be adjusted to aid closure of the LA appendage or a patent foramen ovale and to facilitate concomitant atrial fibrillation ablation. Different part of the annulus may be visualized to allow more accurate placement of annuloplasty sutures. Through a separate working port, which can be as small as 1.5 to $2 \mathrm{~cm}$, the bedside surgeon can actively assist the console surgeon (Figure 1). This LEAR approach (lateral endoscopic approach with robotics) as championed by Dr Murphy can reproduce accurately and efficiently the time tested open mitral repair techniques to achieve both durable results and quick patient recovery. ${ }^{1}$

\section{OUR APPROACH TO ROBOTIC MV REPAIR}

It is our belief that the ability to tailor the repair to different patient circumstances, rather than a "one-size fits all" approach, will result in high rates of the ideal repair. The ideal repair is one in which there is no MR, no mitral stenosis, and a significant coaptation surface below the level of the mitral annulus with low stress forces on the chordae and the leaflets. A typical repair for degenerative posterior leaflet disease in our hands through the robotic approach would be as follows:

1. Triangular resection and repair

2. Polytetrafluoroethylene neochords to the posterior leaflet

3. Cleft repair

4. Delamination of the anterior leaflet

5. Complete annuloplasty ring

The aim of the first step is not to repair the valve but to balance the volume of anterior and posterior leaflet tissue. Step 2 addresses excess leaflet movement, whereas step 3 is necessary when deep clefts are present to provide support to the adjacent scallop. Step 4 is used when there is scarring of the anterior leaflet (Figure 2). This is common and likely to be related to turbulence and jet lesions. It is like the technique used in rheumatic mitral repair. It increases the length, width, and mobility of the anterior leaflet (Video 1). Using an annuloplasty sizer, we have demonstrated that in most patients the area of the anterior leaflet can be increased by up to one size.

The same sequence is performed by the authors in the open sternotomy approach. The need for individual components is tailored to the patient's pathology. The robotic platform allowed the exact replica of these techniques without compromise due to limited access. In addition, due to the lack of distortion of the MV from the lateral approach, 


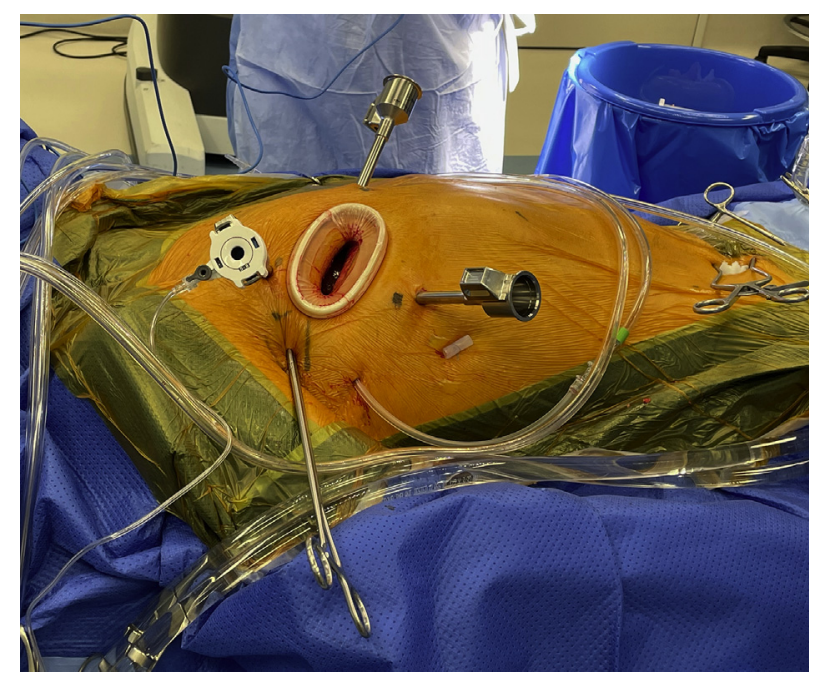

FIGURE 1. Setup for robotic case with combined camera and working port.

the saline static test is quite predictive of the final repair morphology and fine tuning of the repair is facilitated. This strategy has evolved over 17 years of experience with robotic MV repair, with the performance of approximately 40 cases a year at the authors' institution. It has enabled a moderate-volume operator to achieve a repair rate for degenerative disease greater than $99 \%$, with low complications and a mortality less than $0.2 \%$ (Aubrey Almeida, unpublished data, 2021).

However, as many expert mitral surgeons would readily acknowledge, a simple P2 flail in an otherwise-fit and well patient can be performed highly effectively irrespective of approach. However, given the advantages of robotic approach, does it offer any benefit for greater-risk patients with more complex pathology?

\section{ROBOTIC APPROACH FOR COMPLEX MITRAL PATHOLOGY}

A number of meta-analyses have shown that minimally invasive MV repair in elderly ( $>65$ years $)^{8}$ and high risk-patients ${ }^{9}$ had comparable results with those via the median sternotomy approach. Loulmet and colleagues ${ }^{10}$ presented their data on 64 patients with mitral annular calcification undergoing robotic mitral surgery, which accounted for just less than $13 \%$ of their robotic case volume over a 6year period. The authors reported a $97 \%$ MV repair rate with none with more than trace or mild MR at discharge. There was no stroke, and the 30-day mortality was 3.1\%. The same authors also analyzed their entire robotic mitral experience over the same period in which $24 \%$ (of 500) was considered most complex (bileaflet repair or atrioventricular groove reconstruction for mitral annular calcification) and concluded that despite longer crossclamp time and cardiopulmonary bypass time, the patient outcomes have not worsened despite having more complex repairs. ${ }^{11}$ Similarly, Fujita and colleagues ${ }^{12}$ presented 9-year data on 335 patients undergoing either robotic or a minithoracotomy approach; $17 \%$ and $4 \%$ of the repairs, respectively, were considered most complex and the robotic approach was associated with excellent early outcomes.

What exactly constitutes a complex repair is also debatable. Repair of bileaflet prolapse is now routine in experienced hands, regardless of the surgical approach. Perhaps a greater challenge is the situation of a deficiency of leaflet tissue and that of abnormal ventricular size and shape. The robotic approach offers improved vision, lack of distortion of the MV, and instruments that function at the tissue plane should provide surgeons with an advantage in these cases. The ability to increase the leaflet area by delamination and make subtle adjustments under accurate vision does enable these valves to be successfully repaired. It is up to the surgeon to use the equipment to optimize patient outcomes.

\section{BARRIERS IN ROBOTIC MV SURGERY}

In our opinion, the robotic platform is an adaptive and enabling technology with important advantages over other minimally invasive approaches. However, we would also readily acknowledge that these excellent outcomes are largely based on high-volume centers and that there are definite barriers to achieve the full potential of the robotic approach.

The financial aspect of robotic MV surgery is often raised as a concern. Yet, with care, additional costs can be kept to a minimum. As many hospitals already have robotic systems available for other specialties, it is often unnecessary to purchase an additional robotic system. A comparison of 107 robotic mitral repairs to 40 conventional mitral repairs performed at our institution demonstrated a significant reduction in intensive care and hospital stay with a small increase in hospital costs but a reduction in societal cost. ${ }^{13}$ However, enabling a greater rate of repair is a significant cost advantage when one considers the cost of a prosthetic valve and the cost of lifelong anticoagulation. The robotic console surgeon must not only be facile with the equipment but also have knowledge of how to repair the MV. The robotic approach only facilitates what the surgeon can achieve through open repair. Just as not every surgeon is an expert mitral surgeon, the robotic platform is not for every program. There are technical procedures such as peripheral bypass, techniques of myocardial protection, and setup principles such as patient positioning and port placement that must be mastered. These can be overcome with appropriate training with a learning curve that is real but surmountable.

Perhaps the highest hurdle is ensuring that there is dedicated and well-trained team. The roles of anesthesiologist, bedside surgeons, and nursing and technical staff are far more critical than in other operations. ${ }^{14}$ If one is unable to 


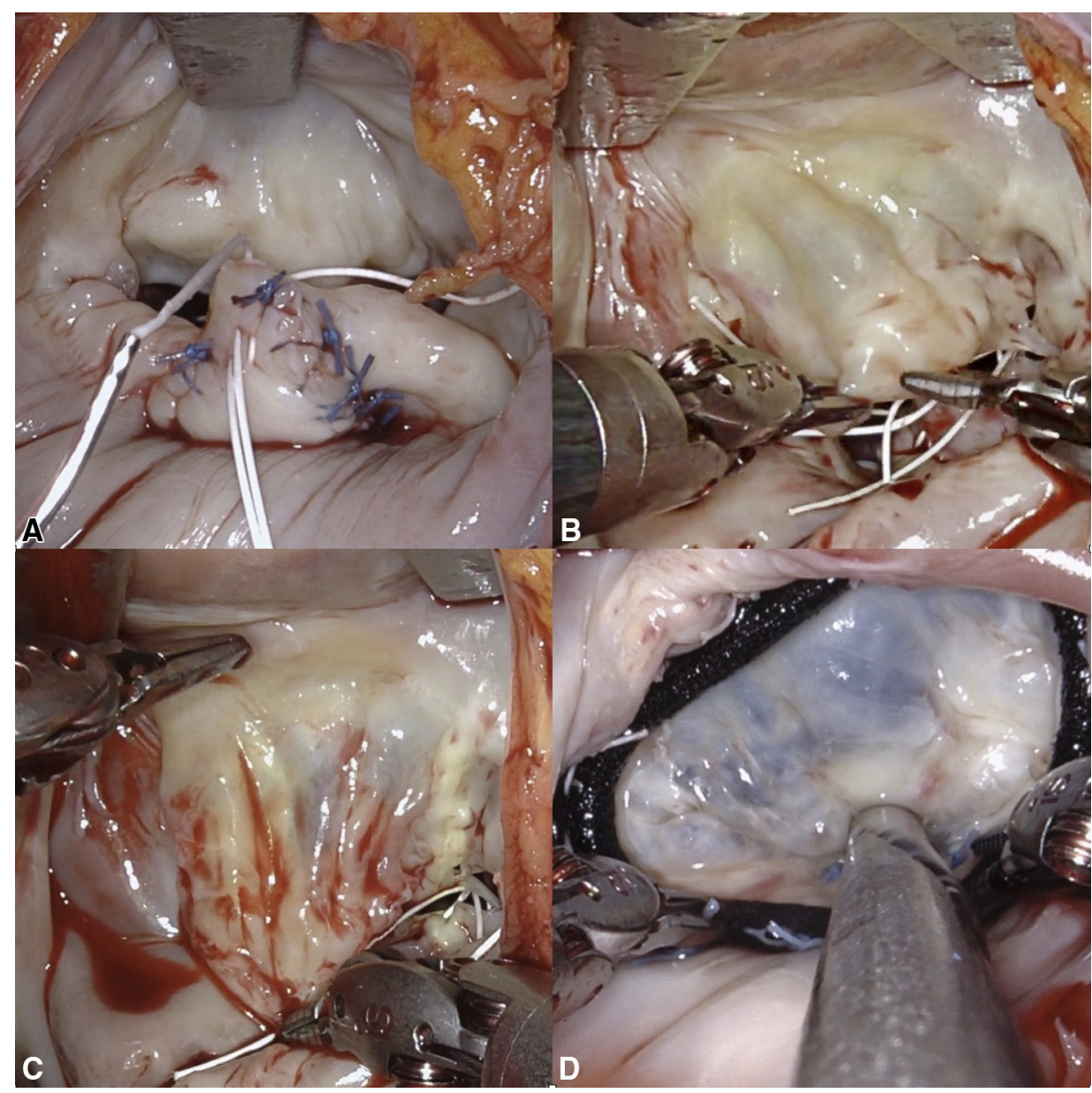

FIGURE 2. Steps of robotic mitral valve repair. A, Triangular resection, cleft closure, and polytetrafluoroethylene neochord. B, Anterior leaflet before delamination. C, Anterior leaflet following delamination, demonstrating increase in surface area. D, Saline test post MV repair and annuloplasty, demonstrating near-normal translucency of anterior leaflet.

arrange and keep this dedicated team together, there is little chance of a successful program.

In starting a robotic program, patient selection is key and should be adjusted to expertise of the team. ${ }^{15}$ Inherently, a robotic operation will have longer bypass and total operating times, as there are more steps than in a sternotomy approach. Consideration should be given to this in patients with severe left ventricular or right ventricular dysfunction. Other cases to avoid are those with significant aortoiliac disease and those with heavy mitral annular calcification. ${ }^{14}$ Only when the team has gone through the initial learning curve should it embark on performing procedures on more complex and high-risk patients, in whom the robotic approach probably offers the most benefit. In this sense, robotic mitral surgery may not be the "best" approach for every mitral surgeon, but it may be the most versatile approach for a dedicated mitral surgery team wanting to offer high-quality repair to patients across the entire spectrum of risks.

\section{CONCLUSIONS}

There is no question that patients are looking for lessinvasive options to treat heart disease than traditional

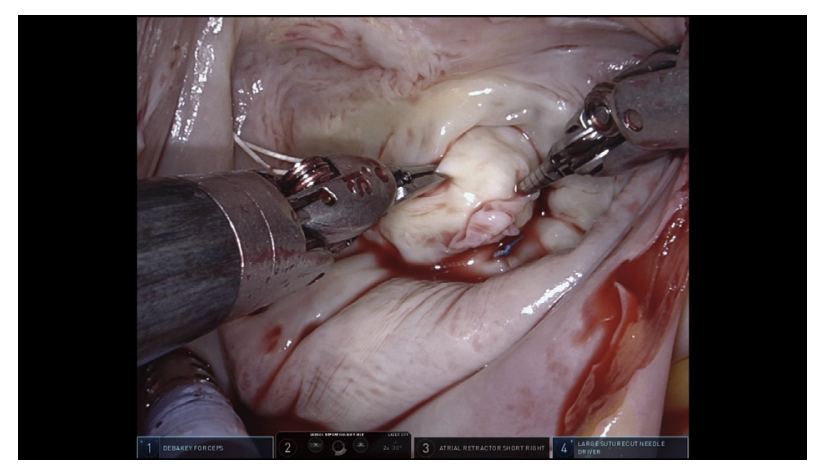

VIDEO 1. Delamination of anterior leaflet as part of degenerative mitral valve repair. Video available at: https://www.jtcvs.org/article/S26662507(21)00686-6/fulltext. 
cardiac surgery. Percutaneous mitral procedures are increasingly offered to treat MR in high-risk and elderly patients, and it is likely this will expand to a wider population. It is critical that surgeons can perform MV surgery in a manner that is most acceptable to patients without any compromise to providing the greatest quality repair. There will always be a disagreement regarding the best approach to minimally invasive MV repair. Good evidence to support one approach over another is currently not available. However, the robotic platform is sophisticated enabling technology, which should help optimize MV repair. It is up to surgeons to ensure the equipment is used in a manner that fulfills this potential.

We believe a Centre of Excellence in MV procedures must be able to perform the spectrum of procedures from percutaneous, to robotic and open surgery. This will enable the best choice to be made considering the patient's risk profile, anatomical suitability and personal needs.

\section{Conflict of Interest Statement}

The authors reported no conflicts of interest.

The Journal policy requires editors and reviewers to disclose conflicts of interest and to decline handling or reviewing manuscripts for which they may have a conflict of interest. The editors and reviewers of this article have no conflicts of interest.

\section{References}

1. Murphy DA, Moss E, Binongo J, Miller JS, Macheers SK, Sarin EL, et al. The expanding role of endoscopic robotics in mitral valve surgery: 1,257 consecutive procedures. Ann Thorac Surg. 2015;100:1675-81; discussion 81-2.

2. Gillinov AM, Mihaljevic T, Javadikasgari H, Suri RM, Mick SL, Navia JL, et al. Early results of robotically assisted mitral valve surgery: analysis of the first 1000 cases. J Thorac Cardiovasc Surg. 2018;155:82-91.e2.
3. Castillo JG, Anyanwu AC, Fuster V, Adams DH. A near 100\% repair rate for mitral valve prolapse is achievable in a reference center: implications for future guidelines. J Thorac Cardiovasc Surg. 2012;144:308-12.

4. Cao C, Wolfenden H, Liou K, Pathan F, Gupta S, Nienaber TA, et al. A metaanalysis of robotic vs. conventional mitral valve surgery. Ann Cardiothorac Surg. 2015;4:305-14.

5. Nair RU, Sharpe DA. Limited lower sternotomy for minimally invasive mitral valve replacement. Ann Thorac Surg. 1998;65:273-4.

6. Glauber M, Miceli A, Canarutto D, Lio A, Murzi M, Gilmanov D, et al. Early and long-term outcomes of minimally invasive mitral valve surgery through right minithoracotomy: a 10-year experience in 1604 patients. J Cardiothorac Surg. 2015;10:181.

7. Sa M, Van den Eynde J, Cavalcanti LRP, Kadyraliev B, Enginoev S, Zhigalov K, et al. Mitral valve repair with minimally invasive approaches vs sternotomy: a meta-analysis of early and late results in randomized and matched observational studies. J Card Surg. 2020;35:2307-23.

8. Hage A, Hage F, Al-Amodi H, Gupta S, Papatheodorou SI, Hawkins R, et al. Minimally invasive versus sternotomy for mitral surgery in the elderly: a systematic review and meta-analysis. Innovations (Phila). 2021;16:310-6.

9. Moscarelli M, Fattouch K, Casula R, Speziale G, Lancellotti P, Athanasiou T. What is the role of minimally invasive mitral valve surgery in high-risk patients? A meta-analysis of observational studies. Ann Thorac Surg. 2016; 101:981-9.

10. Loulmet DF, Ranganath NK, Neragi-Miandoab S, Koeckert MS, Galloway AC Grossi EA. Advanced experience allows robotic mitral valve repair in the presence of extensive mitral annular calcification. J Thorac Cardiovasc Surg. November 2, 2019 [Epub ahead of print].

11. Loulmet DF, Ranganath NK, Neuburger PJ, Nampiaparampil RG, Galloway AC Grossi EA. Can complex mitral valve repair be performed with robotics? An institution's experience utilizing a dedicated team approach in 500 patients. Eur J Cardiothorac Surg. 2019;56:470-8.

12. Fujita T, Kakuta T, Kawamoto N, Shimahara Y, Yajima S, Tadokoro N, et al. Benefits of robotically-assisted surgery for complex mitral valve repair. Interact Cardiovasc Thorac Surg. 2021:32:417-25.

13. Kam JK, Cooray SD, Kam JK, Smith JA, Almeida AA. A cost-analysis study of robotic versus conventional mitral valve repair. Heart Lung Circ. 2010;19:413-8.

14. Suri RM, Dearani JA, Mihaljevic T, Chitwood WR Jr, Murphy DA, Trento A, et al. Mitral valve repair using robotic technology: safe, effective, and durable. J Thorac Cardiovasc Surg. 2016;151:1450-4.

15. Tang RC, Murphy DA, Moss E. Choosing the ideal candidate for a robotic valve intervention. Can J Cardiol. 2021;37:1117-20.

Key Words: mitral valve, repair, robotic, minimally invasive 